\title{
Introducing HCI in Corporate IT Department in a Large Company in Brazil
}

\author{
Andre Vinicius Fontes Dantas ${ }^{1}$, Carlos Freud Alves Batista ${ }^{1}$, Cassiano Ebert ${ }^{1}$, \\ Maíra Greco de Paula ${ }^{1,2}$, and Simone Diniz Junqueira Barbosa ${ }^{2}$ \\ ${ }^{1}$ Petrobras/TI/SERV-TI/DS, Rua da Assembléia, 100, Centro, RJ, Brasil \\ ${ }^{2}$ Informatics Dept., PUC-Rio, Rua Marquês de São Vicente, 225, Gávea, RJ, Brasil \\ \{andrevinicius, carlos.freud, cassiano.ebert, \\ maira.greco.STK\} @petrobras.com.br, simone@inf.puc-rio.br
}

\begin{abstract}
This paper describes the introduction of human-computer interaction activities in the Corporate IT Department in a large energy company in Brazil. It is certified by ISO 9001:2000, and thus has a set of norms that IT employees must follow during the software development process. We discuss the introduction of $\mathrm{HCI}$ activities into these norms.
\end{abstract}

Keywords: software development process norms, human-computer interaction.

\section{Introduction}

This paper describes the introduction of human-computer interaction (HCI) activities in the Corporate Information Technology (IT) Department in a large energy company in Brazil, which develops software for the company employees. It is certified by ISO 9001:2000, and thus has a set of norms that IT employees must follow during the software development process. For instance, there are norms for the development of applications following certain methodologies, and for software inspection and testing.

Due to the great diversity of users and system domains, we have decided to create a norm regarding the application of HCI concepts during the software development process. To develop this norm, a software engineering-usability technical group was formed with IT employees from various software development regions who had interest or experience in HCI. Before writing the norm, the group members participated in a tutorial on HCI, given by an R\&D HCI laboratory ${ }^{1}$. This tutorial presented an overview of HCI concepts and design and evaluation activities. Also, the group members analyzed the software development standards in the company and then proposed an HCI application norm for the corporate IT.

\section{The Development Process and the HCI Application Norm}

The software development process in our company is composed of the following activities: 0) request from a business area; 1) process definition; 2) requirements analysis; 3) software design (logical and physical); 4) software construction, making

\footnotetext{
${ }^{1}$ SERG at PUC-Rio: http://www.serg.inf.puc-rio.br
} 
use of available frameworks and components; 5) software testing; 6) software validation with the client; and 7) deployment. There are standards guiding each activity and the construction of each artifact in the software development process. Also, inspection activities occur in parallel with the design and construction stages.

The HCI norm introduced new activities and artifacts in this process (Table 1) [1]. We highlight here the user interface (UI) model, which is composed of a) wireframes; b) the associated requirements; c) a description of each screen and UI element, indicating which ones are mandatory or optional, the default values, and data format, when applicable; d) relationships or dependencies between the UI elements; and e) a navigation map between the various screens. In its first version, the norm is applicable only to web applications. Usability inspection activities were introduced in two stages: during software design, of both UI model and prototype (if available); and after the software construction, of the final product. Some activities were defined as optional due to the lack of maturity and available professionals to carry them out.

Table 1. Usability-related activities introduced by the norm

\begin{tabular}{lll}
\hline Sw Dev Stage & HCI-related activity & Type \\
\hline Requirements analysis & UI requirements elicitation questionnaire & Optional \\
Software design & UI model or & Mandatory \\
& UI prototype construction & \\
Software design & UI model/prototype inspection & Mandatory \\
Software construction & Final product's UI inspection & Mandatory \\
Software testing & Usability testing & Optional \\
\hline
\end{tabular}

\subsection{Pilot Project}

The user interface of a business-critical system was redesigned according to the new HCI norm, and presented to the higher IT management. The result was very positive, and everyone agreed with having a usability-related norm. Therefore, the technical group gained management support, which is crucial in a large company with IT departments distributed in various regions. Some developers and graphical designers commented that they liked the UI model very much, in that they didn't need to make decisions about HCI when doing their work.

\subsection{Training and Deployment of the HCI Norm}

Several employees were trained, including project leaders, analysts, developers, inspectors and testers. The inspectors showed some resistance, because they now have additional artifacts to inspect, besides all the software design artifacts traditionally used. We expect the changes in the organizational culture to be gradual, and will keep monitoring the professionals' attitudes towards the norm to make the necessary adjustments in the HCI-related activities or roles that perform them. The technical group is now monitoring the norm's application in the various regional departments, and is also extending the norm to include user interfaces for websites and portals, desktop application, Lotus Notes ${ }^{\circledR}$ applications, and mobile devices. 
Acknowledgments. We thank all the members of the usability technical group in our company for their work in the usability standards and norms described here.

\section{References}

1. Solutions Development Area at PETROBRAS Corporate IT Department, Usability Guidelines for the Construction of Web User Interfaces, TI-IT-1T1-00123 (2006) 